\title{
Operation of an optical in-well-pumped vertical-external-cavity surface-emitting laser
}

\author{
Wei Zhang, Thorsten Ackemann, Stephen McGinily, Marc Schmid, Erling Riis, \\ and Allister I. Ferguson
}

\begin{abstract}
We report the operation of an optical in-well-pumped vertical-external-cavity surface-emitting laser. The laser delivers $1 \mathrm{~W}$ at $855 \mathrm{~nm}$ and is pumped with a cost-effective fiber-coupled laser diode emitting at $806 \mathrm{~nm}$. The laser modal gain is examined and ways of optimizing the system are investigated and discussed. (C) 2006 Optical Society of America

OCIS codes: $140.3480,140.5960,140.5560,230.5590,250.7260$.
\end{abstract}

\section{Introduction}

Optically pumped vertical-external-cavity surfaceemitting lasers (VECSELs) have been studied extensively for several years, ${ }^{1-3}$ and commercial products based on this technology are available. With only a few exceptions, the vast majority of the research in this area makes use of quantum-well (QW) gain structures based on InGaAs. These VECSELs usually emit light in the region between 950 and $1100 \mathrm{~nm}$. Laser diodes operating around $800 \mathrm{~nm}$ are used as pump sources. These are inexpensive and powerful commercial devices, well known for pumping high-power Nd:YAG lasers. The current record of high-power cw optically pumped VECSELs was achieved with an InGaAsbased system producing $30 \mathrm{~W}$ at $980 \mathrm{~nm} .^{4}$

VECSELs at shorter wavelengths (840-860 nm; $670 \mathrm{~nm}$ ) are known to have much lower output power; the maximum reported values are $0.5 \mathrm{~W}$ for $850 \mathrm{~nm}$ (Ref. 5) and $1 \mathrm{~W}$ for a $670 \mathrm{~nm}$ laser. ${ }^{6}$ One of the main problems with these systems is the availability of a suitable pump source. For example, VECSEL systems based on GaAs QWs, emitting at $~ 850 \mathrm{~nm}$ are normally pumped with laser diodes emitting below $700 \mathrm{~nm}$. However, these pump sources are significantly more expensive and less bright than their counterparts at $\sim 800 \mathrm{~nm}$. As a consequence, GaAsbased VECSELs are much less powerful than the

The authors are with the Department of Physics, University of Strathclyde, Glasgow G4 ONG, Scotland, U.K. W. Zhang's e-mail address is wei.zhang.100@strath.ac.uk.

Received 24 May 2006; accepted 24 May 2006; posted 30 May 2006 (Doc. ID 71141).

0003-6935/06/297729-07\$15.00/0

(C) 2006 Optical Society of America equivalent systems based on InGaAs. In this paper, we report an optically pumped GaAs-based system emitting in the $850 \mathrm{~nm}$ region with an output power exceeding $1 \mathrm{~W}$ but pumped with an $806 \mathrm{~nm}$ diode laser. Such a laser is interesting for many applications requiring moderately high power in a single longitudinal and transverse mode. Here we are particularly interested in the applications for laser cooling, such as laser cooling of cesium. ${ }^{7}$ These characteristics are of even greater importance for a system from which a frequency-doubled output is required, e.g., as used in laser cooling of chromium or calcium. ${ }^{8,9}$

We have already demonstrated the concept of in-well pumping of a VECSEL in the $850 \mathrm{~nm}$ wavelength region. ${ }^{10}$ In this paper, we explore the powerscaling aspect of this technique. The main difference in pumping around $800 \mathrm{~nm}$ instead of below $700 \mathrm{~nm}$ is that the barrier region between the QWs is now transparent. Therefore the pump light at $800 \mathrm{~nm}$ is only absorbed in the QWs themselves (in-well pumping), and we estimate this absorption to be a mere $1 \%$ per QW per single pass. ${ }^{11,12}$ In contrast, a gain structure designed and optimized for conventional barrier pumping (below $700 \mathrm{~nm}$ ), where the carriers are created between and subsequently drift into the QWs, has a typical absorption of $10 \%$ per barrier. The gain structure used for the present investigations was designed for barrier pumping and has only $17 \mathrm{QWs}$. Hence only a relatively small fraction of the $800 \mathrm{~nm}$ pump light is absorbed per pass through the gain region. However, in this paper we demonstrate that it is possible to increase the total absorption of the pump and hence increase the total output power of this type of system by carefully choosing the pump wavelength and adapting the pump geometry. We also discuss the optimization of the gain element. 
In-well pumping has further advantages over conventional barrier pumping than just allowing the use of powerful $800 \mathrm{~nm}$ diodes to pump $850 \mathrm{~nm}$ VECSELs. As the quantum efficiency is increased, less pump power is converted into heat, and hence the VECSEL can be pumped harder. This aspect has recently been explored further in the context of the in-well pumping of a $980 \mathrm{~nm}$ laser with a $940 \mathrm{~nm}$ pump. ${ }^{13}$ Furthermore, in barrier pumping, the absorption length of the barrier turns the number of QWs into a critical design parameter. Too few wells will result in a significant fraction of the expensive $700 \mathrm{~nm}$ pump light being transmitted through to and absorbed in the Bragg stack and substrate below the gain region. If there are too many $\mathrm{QWs}$, the last ones will not be pumped sufficiently and effectively act as absorbers at the VECSEL wavelength. In in-well pumping, there is, in practice, no such limit. We are willing to accept that a significant fraction of the inexpensive $800 \mathrm{~nm}$ pump light is not absorbed. VECSEL gain element designs usually include a Bragg stack immediately below the $\mathrm{QW}$ region, which is highly reflecting for the laser wavelength. This Bragg stack can also be designed to be highly reflecting for the pump light, allowing it to pass through the QW region once more and enabling the recycling of any nonabsorbed pump light similar to what has been demonstrated for the thin-disk solid-state laser. ${ }^{14}$ Finally, in-well pumping offers the possibility of extending the wavelength coverage of diode-laserpumped VECSELs well below the $850 \mathrm{~nm}$ region. For example, fiber-coupled laser diodes around $670 \mathrm{~nm}$ with an output power of several watts could be used to pump VECSELs emitting around $700 \mathrm{~nm}$. This is a spectral region that would otherwise be inaccessible with current pump laser technology. More information about in-well pumping can be found in Refs. 10, 13 and 15.

Apart from the investigations of optically pumped VECSELs emitting above $950 \mathrm{~nm}$ (Ref. 13), there are only a few reports of lasers operating around 850 and $660 \mathrm{~nm}$ where in-well pumping was used. ${ }^{10,16} \mathrm{In}$ these cases, the achieved output powers were much lower than those reported in this paper. Some reports have been published about the characteristics of in-well pumping of vertical-cavity surface-emitting lasers (VCSELs), but no information is available on power scaling or achieved thresholds. ${ }^{17,18}$ However, they do point out the importance of matching the wavelength with the resonant structure of the microcavity formed by the Bragg stack and the VCSEL surface, similar to the findings in this paper.

\section{Experimental Setup}

The basic structure of the VECSEL gain element used in this work is illustrated schematically in Fig. 1. A semiconductor Bragg mirror was first deposited on top of a GaAs wafer. This high-reflectivity (HR) mirror has its central wavelength at $850 \mathrm{~nm}$ for normal incidence and a bandwidth of $85 \mathrm{~nm}$ FWHM, as shown in Fig. 2. Above the mirror is a $17 \mathrm{QW}$ straincompensated gain structure with a peak emission at

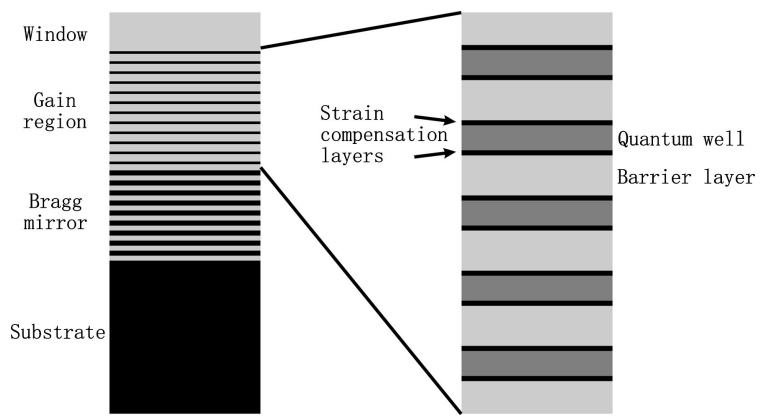

Fig. 1. VECSEL structure. A highly reflecting Bragg mirror and a strain-compensated gain region are grown on a GaAs substrate.

$851 \mathrm{~nm}$. The wells are separated by approximately half a wavelength of AlGaAs. A further layer of AlGaAs is added on top of the gain structure to increase the total length of the microcavity to $2.55 \mu \mathrm{m}$. This corresponds to an antiresonant structure at 850 $\mathrm{nm} .{ }^{19}$ Finally, a $20 \mathrm{~nm}$ layer of GaAsP was added to protect the structure. For all the results presented in this paper, a 500- $\mu$ m-thick uncoated diamond heat spreader was mounted in front of the gain element. ${ }^{20}$ The whole device was clamped between two copper plates and mounted on a water-cooled copper block to control the cooling temperature $T_{C}$ of the VECSEL gain chip. A detailed discussion and characterization of the material will be reported separately. ${ }^{21}$

The laser cavity is formed by the HR Bragg mirror and an external curved output coupler (OC). The radius of curvature of the OC was $150 \mathrm{~mm}$, and the reflectivity was $98 \%$. Owing to the refractive index difference, a fraction of the laser light is reflected at the diamond-semiconductor interface and a microcavity is formed between this surface and the Bragg mirror. Owing to its resonances, this microcavity can significantly enhance the pump absorption efficiency, which is discussed in Section 3. The diamond-air interface causes an approximately $17 \%$ reflection loss of the pump light. This reflection also causes the diamond heat spreader itself to act as an etalon for the laser resulting in a channeled laser spectrum.

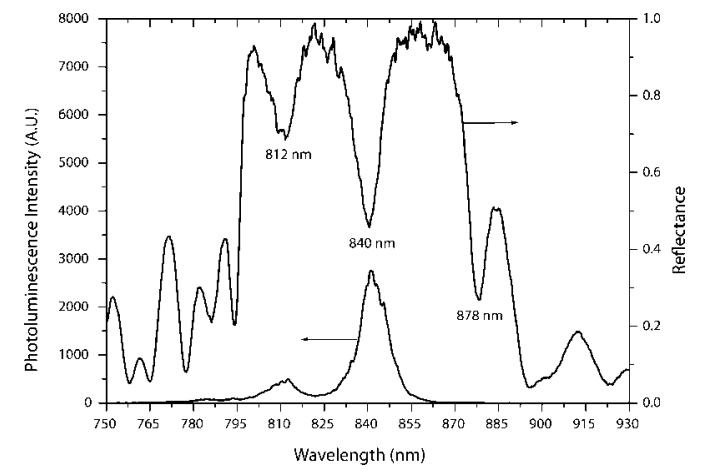

Fig. 2. Measured reflectance (right ordinate) and photoluminescence emission spectra (left ordinate) of the VECSEL sample at room temperature with normal incidence. The sample is excited by an $806 \mathrm{~nm}$ pump source. 
The pump source was operating at $\lambda_{p}=811 \mathrm{~nm}$ at full power and a cooling temperature of $20^{\circ} \mathrm{C}$. By cooling it down to $T_{p}=-6.7^{\circ} \mathrm{C}$, the wavelength dropped to $\lambda_{p}=806 \mathrm{~nm}$, while the output power did not change significantly. The laser was coupled into a $100 \mu \mathrm{m}$ fiber with a NA of 0.22 . After the focusing system, the spot size on the VECSEL was roughly $110 \mu \mathrm{m}$ in diameter. The length of the external cavity was approximately $149 \mathrm{~mm}$. Different pump incident angles were investigated to search for the optimum angle for best pump absorption at the specific pump wavelength. After a single pass through the gain region, the remaining pump light was reflected by the HR Bragg mirror and passed through the gain region a second time. The remaining pump power, approximately $65 \%$, which was not absorbed after the double pass, could be reflected back onto the VECSEL with a curved gold-coated mirror. With this additional recycling loop, approximately $92 \%$ of the nonabsorbed pump power could be launched in a second double pass through the VECSEL. However, of this, $17 \%$ will again be lost at the diamond-air interface. These losses could be greatly reduced from the present total of $34 \%$ to less than $5 \%$ by use of antireflective (AR) coatings at the diamond heat spreader at pump wavelength.

\section{Results and Discussion}

\section{A. Reflectivity and Photoluminescence}

Before building the external cavity, the reflectance and photoluminescence (PL) spectrum of the gain medium was recorded for normal incidence, as shown in Fig. 2. From this, we can clearly identify the positions and strengths of the resonances for pumping and lasing as well as the position of the Bragg reflectance band. The observed PL is a function of the QW emission, and the spectral filtering caused by the microcavity resonances described above results in the main peak of the PL signal, which matches exactly with one of the absorption enhancement resonances. Due to the antiresonant design of this wafer, the designed laser wavelength (approximately $850 \mathrm{~nm}$ and defined by the resonant periodic gain) lies in the range between two resonances (840 and $878 \mathrm{~nm}$ ).

The resonances of the semiconductor structure are also found to be of importance in enhancing the pump absorption in the gain medium. ${ }^{10,13}$ When the cavity formed between the Bragg mirror and the VECSEL surface is resonant for a particular pump wavelength, the pump intensity will build up, resulting in an increased absorption. This not only leads to a sensitivity to the pump wavelength for the in-wellpumping scheme, which is much higher than for conventional barrier pumping, ${ }^{1}$ but also leads to a significant dependence on the pump angle.

$\mathrm{PL}$ is a direct result of $\mathrm{QW}$ carriers being generated by the pump light and hence a good measure for the absorption in the structure. We investigated the PL excitation spectra for different pump angles to find the optimum pump wavelength for a given incident angle. Figure 3 shows the PL excitation spectra of the

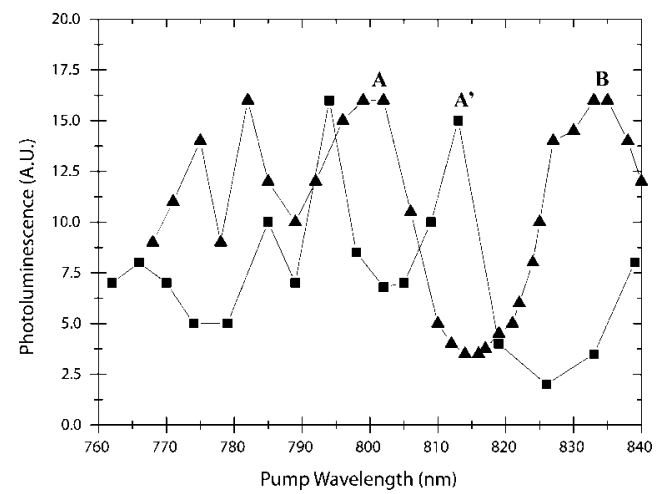

Fig. 3. PL excitation spectrum. The peak fluorescence at around $840 \mathrm{~nm}$ is shown as a function of the excitation wavelength. The angle of incidence is near $0^{\circ}$ for the square symbols and $45^{\circ}$ for the triangular symbols, respectively. The operating temperature was $20^{\circ} \mathrm{C}$.

VECSEL sample for near-normal incidence and at a $45^{\circ}$ angle of incidence for the pump light. For this experiment, a tunable $\mathrm{cw}$ Ti:sapphire laser was used as a pump source. The PL was detected perpendicular to the VECSEL surface by using a multimode fibercoupled CCD spectrometer ( $0.2 \mathrm{~nm}$ resolution). Points $\mathrm{A}$ and $\mathrm{A}^{\prime}$ correspond to the same maximum but are separated by $15 \mathrm{~nm}$. This is the expected shift of resonance for a propagation angle of $11.7^{\circ}$ inside the gain medium, which agrees well with the $45^{\circ}$ angle of incidence. Points A and B are the two maxima of the microcavity separated by a free spectral range (FSR) of $16.8 \mathrm{THz}$ corresponding to the $2.55-\mu \mathrm{m}$-thick gain structure. It has to be noted that a wavelength shorter than $790 \mathrm{~nm}$ for the $45^{\circ}$ curve and $810 \mathrm{~nm}$ for the normal incidence curve of the Bragg mirror is no longer highly reflecting and the behavior of the microcavity is more complicated.

Figure 3 indicates that the excitation is not optimum in the wavelength range of our current pump source $(806-811 \mathrm{~nm})$ and for an angle of incidence of $45^{\circ}$. To match the pump wavelength to a microcavity resonance, the pump angle must be reduced. A more detailed investigation on the optimum pump angle was carried out in the wavelength region of 800$810 \mathrm{~nm}$. The tunable cw Ti:sapphire laser was again used as the pump source. The laser beam was focused onto the sample to give a spot size of roughly $500 \mu \mathrm{m}$ in diameter. The sample was mounted on a rotation stage, which can provide a $360^{\circ}$ rotation with the axis parallel to the front surface of the sample. For each wavelength, the sample was rotated and the reflected power and normal PL intensity were monitored simultaneously. The angle at which the PL was maximized was recorded for a range of wavelengths. Figure 4 shows these optimum angles. The optimum angle decreases as the wavelength increases, which is consistent with the data shown in Fig. 3. The reason for this is that the periodicity of the standing-wave pattern of the pump radiation along the cavity axis can be related to the pump wavelength $\lambda_{p}$ by the expression $\lambda_{\mathrm{swp}}=\lambda_{p} / \cos \left(\Theta_{\mathrm{in}}\right)$. By changing the inci- 


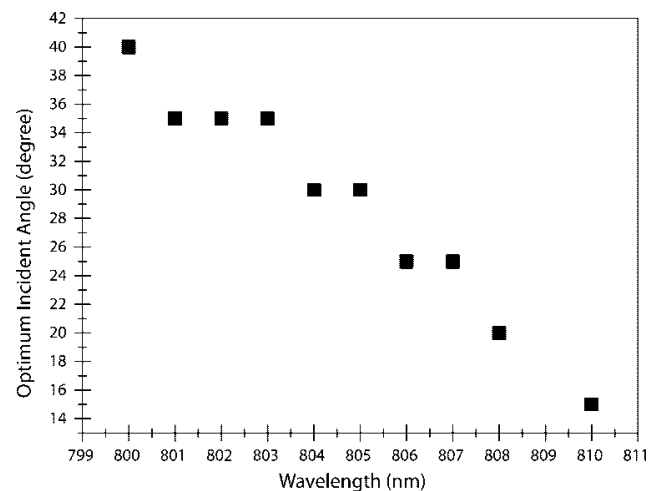

Fig. 4. Optimum pump angle as a function of pump wavelength. The optimum pump angle was taken to be that which resulted in the highest PL intensity at the $840 \mathrm{~nm}$ maximum. The operating temperature was $20^{\circ} \mathrm{C}$.

dent pump angle so as to change the internal angle of propagation $\Theta_{\text {in }}$, the periodicity of the standing-wave pattern can be tuned to match the microcavity resonance $(812 \mathrm{~nm})$, as shown in Fig. 2. The optimum angle is around $27^{\circ}$ for $806 \mathrm{~nm}$, decreasing to approximately $15^{\circ}$ at $810 \mathrm{~nm}$. Due to the mechanical limitation of our system, we have to operate with a pump angle larger than $20^{\circ}$. The data presented on highpower operation in this paper were taken with an $806 \mathrm{~nm}$ pump incident at approximately $27^{\circ}$.

The high sensitivity of the laser absorption on the pump wavelength also indicates that at all pump powers, the operating temperature of the pump should be adapted to keep the diode emission wavelength fixed. For laser operation, an external mirror with $R_{C}=150 \mathrm{~mm}$ is aligned to complete the laser cavity. While keeping the pump constant (both wavelength and power) and only changing the temperature of the mount onto which the gain element was attached, the optimum laser cooling temperature $T_{C}$ at maximum pump power was found to be $20{ }^{\circ} \mathrm{C}$. The actual QW region of the gain element will be operating at a significantly higher temperature due to the thermal gradients across the semiconductor material, 22 and we can therefore expect $T_{c}$ to depend on the heat load (absorbed pump power) of the system.

\section{B. High-Power Operation}

After setting the pump angle to be $\sim 27^{\circ}$ and the laser cooling temperature to $20^{\circ} \mathrm{C}$, a maximum power of $380 \mathrm{~mW}$ was obtained. The laser was clearly operating with higher-order transverse modes. The reflected pump light was then imaged back onto the gain element using a gold-coated curved mirror. This pump recycling provided an additional double pass through the QW gain region and resulted in an increase of the laser power by a factor of 2.5. The additional heat load on the gain medium resulted in a drop of the optimum laser cooling temperature to $15{ }^{\circ} \mathrm{C}$.

The output power of the VECSEL as a function of the pump power with and without recycling of the pump light back to the gain medium was investigated

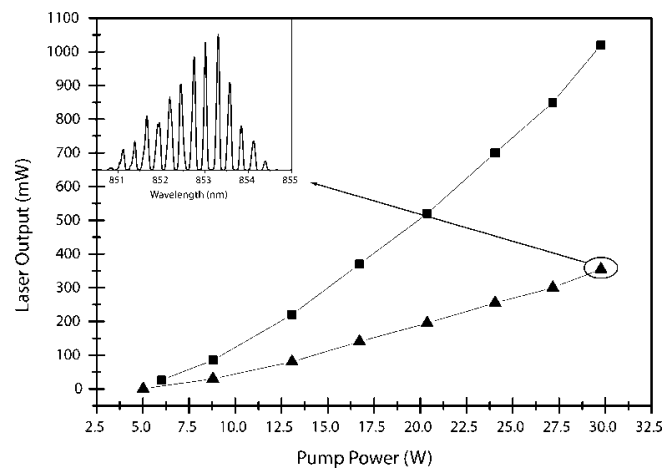

Fig. 5. Laser output power versus pump power at a pump angle of $27^{\circ}$ and a laser temperature of $15^{\circ} \mathrm{C}$ with (upper curve with square symbols) and without (bottom curve with triangular symbols) retroreflection. The pump wavelength was fixed at $806 \mathrm{~nm}$ by changing the pump cooling temperature at different powers. Inset, VECSEL spectrum at maximum pump power and no recycling. The structure in the spectrum is due to the diamond heat spreader acting like an etalon.

and is shown in Fig. 5. Also shown is an example of the output spectrum of the laser. The individual peaks in this channeled spectrum are separated by the FSR of the diamond. Suppression of this etalon is possible using an AR-coated and wedged heat spreader. ${ }^{23}$

A maximum output power of $1.02 \mathrm{~W}$ at $855 \mathrm{~nm}$ was achieved at a pump power of $29.8 \mathrm{~W}$, which corresponds to an estimated absorbed power of $12.5 \mathrm{~W}$; see also Fig. 6. As indicated above, the pump wavelength was kept constant at $806 \mathrm{~nm}$. The absorption in the QW region was estimated from measurements of the pump reflectivity. After subtracting the constant reflection loss (17\%) at the front surface of the diamond for each pass, the double-pass absorption of the VECSEL without retroreflection and at maximum pump power $(29.8 \mathrm{~W})$ was $\sim 30 \%$. With retroreflection in place, $45 \%$ of the total incident pump power was reflected back onto the gain medium. Based on measurements of an increase in the absorption with increasing pump power due to the heating of the gain region, we estimate the overall double-pass absorp-

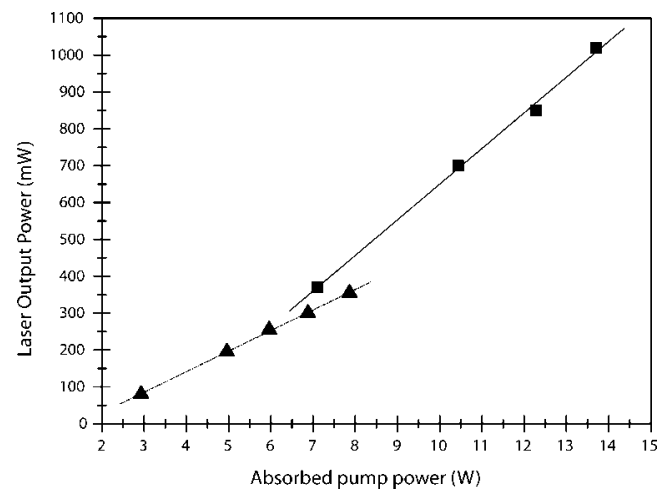

Fig. 6. Laser output power versus absorbed pump power for the laser with (square symbols) and without (triangular symbols) pump retroreflection. The slope efficiencies for the laser without and with pump recycling are $6 \%$ and $10.6 \%$, respectively. 


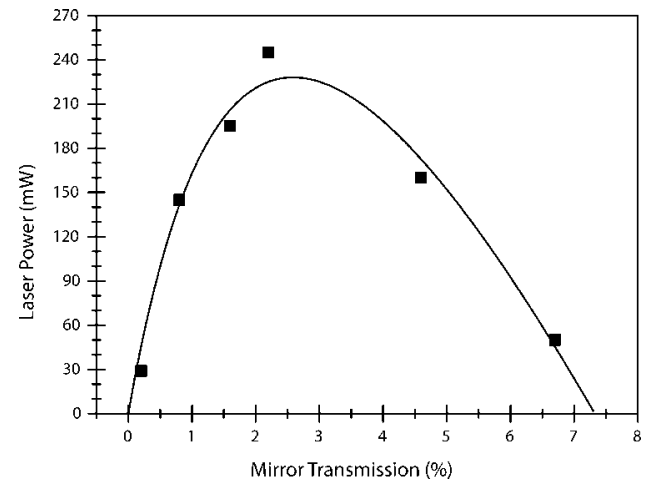

Fig. 7. Output power versus output coupling without pump retroreflection. The fitted curve (see text) indicates a gain of $10.4 \%$ and an internal loss of $3.1 \%$ in the cavity.

tion to be $\sim 35 \%$. It is apparent from both sets of data that the output power increases more rapidly than the normal linear dependence on pump power. This is because the pump wavelength and laser temperature were optimized for the highest power level. Since the temperature coefficient of the microcavity resonances is $\sim 0.1 \mathrm{~nm} / \mathrm{K}$, the resonant wavelength shifts to blue when the pump power decreases, and thus laser temperature drops. Hence the pump radiation is detuned gradually from the resonance generating the concave curves. From the shift of resonance with pump power, we estimated a gain-region temperature increase of $50{ }^{\circ} \mathrm{C}$, which is also in accordance with literature values. ${ }^{22}$

If, rather than plotting the laser output power against the incident pump power, we use the additional measurement of the reflected pump power to plot it against the absorbed power, we recover straight line fits. Figure 6 shows this data. The slope efficiencies for the lasers with and without pump retroreflection are determined to be $10.6 \%$ and $6 \%$, respectively. We attribute this difference to the fact that the laser temperature for this data set $\left(15^{\circ} \mathrm{C}\right)$ was optimized for the highest power operation with pump retroreflection. The slope efficiency for in-well pumping was found to be less than the efficiency for barrier pumping $(20 \%-30 \%)$ when the same wafer was used. This can be attributed to the fact that the structure of the wafer is not optimized for in-well pumping. This is discussed in Subsection 3.D.

\section{Laser Gain}

The laser modal gain was examined for the in-wellpumped VECSEL. These measurements were conducted before optimizing the laser system in terms of laser temperature and pump angle. Hence the pump angle was set at $45^{\circ}$, and the laser cooling temperature $T_{c}$ and the pump temperature $T_{p}$ were set to $24^{\circ} \mathrm{C}$ and $-5.5^{\circ} \mathrm{C}$, respectively. With a different focusing system, the pump spot was measured to be approximately $80 \mu \mathrm{m}$ in diameter. To determine the laser gain, the output power of the VECSEL was recorded for a range of output couplers. ${ }^{24}$ Figure 7 shows an example of this for the case of the in-well-

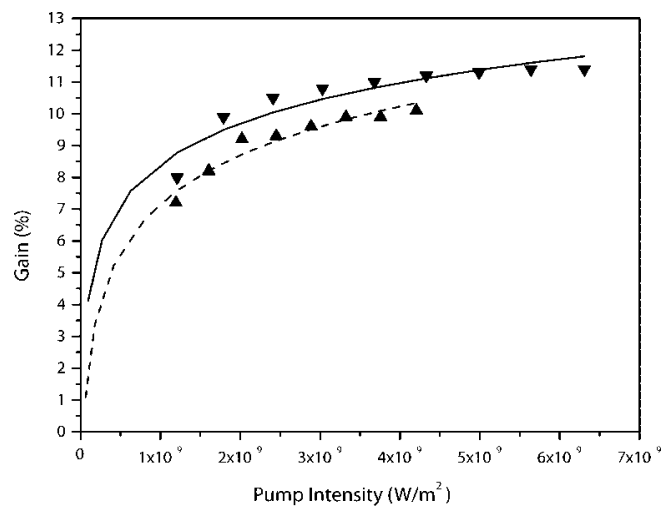

Fig. 8. Gain dependence of the VECSEL with (square symbols fitted with a solid curve) and without (triangular symbols fitted with a dashed curve) pump retroreflection.

pumped VECSEL pumped with $21 \mathrm{~W}$ and without pump recycling. This power corresponds to a pump intensity of $420 \mathrm{~kW} / \mathrm{cm}^{2}$. A fit of the data to the function $^{24} P(T)=C T\left\{\left[g_{0} /\left(L_{i}+T\right)\right]-1\right\}$ yields a value for the laser gain $g_{0}$ of $10.4 \%$ and internal loss $L_{i}$ of $3.1 \%$; the parameters $T$ and $C$ represent the mirror transmission and a constant, respectively. The optimum value for the output coupling, in this case, was determined to be $2.5 \%$, which is close to that of the $2.2 \%$ output coupler used in the experiment.

The gain dependence on pump intensity was also investigated. Values for the modal gain were obtained at different pump powers and plotted against pump intensity. For the cases of the in-well-pumped VECSEL without and with pump recycling, the curves were of the form shown in Fig. 8. Both curves demonstrated a logarithmic dependence, which suggested that the gain dependence of an in-wellpumped VECSEL was similar to that of a barrierpumping one where the gain is logarithmically related to the carrier density. ${ }^{25}$ The value of the gain at a pump intensity level of $630 \mathrm{~kW} / \mathrm{cm}^{2}$ for the laser with retroreflection was taken as around $11.5 \%$. This was an improvement on the $10 \%$ obtained for the laser without pump recycling and suggested enhanced pump efficiency. As shown in Fig. 8, the total set of the data for the laser with retroreflection is slightly higher than the one obtained for the laser without retroreflection. We attribute this difference to the fact that the laser temperature for this data set was optimized for the highest power operation with pump retroreflection.

\section{Discussion}

As seen from the results presented in Fig. 5, there is still significant pump power left even after one recycling loop of the pump light. Additional recycling loops could therefore potentially increase the absorption and hence the total output power of the VECSEL. A multipass pump setup similar to that demonstrated for thin-disk solid-state lasers ${ }^{14}$ would be the preferred geometry for an in-well-pumped system. Because the absorption per single pass in a 
VECSEL structure is higher than in a solid-state thin disk, fewer recycling loops would be required.

To optimize future designs of optically in-wellpumped VECSELs, there are three aspects that have to be taken into account. The first two relate to the resonant structure built into the design of the gain element. They are the intracavity enhancement and the resonant periodic gain (RPG) structure, ${ }^{26}$ which are traditionally only optimized for the laser wavelength. For a conventional barrier-pumping VECSEL device, a standing-wave pattern of the laser electrical field is formed between the front surface of the semiconductor and the Bragg reflector. The QWs are placed at the antinodes of the standing-wave pattern to enhance the intensity of the laser field. This gives an enhancement for the intensity by a factor of $\Gamma_{\mathrm{RPG}}=2$, where $\Gamma_{\mathrm{RPG}}$ represents the enhancement factor for resonant periodic gain, compared to the average intensity. The intensity of the laser field can be further enhanced by the microcavity that is formed between the Bragg reflector and the surface of the structure. This can be designed to be resonant at the laser wavelength to yield a VECSEL with a particularly low threshold. For barrier pumping, it is not necessary to consider any interference effects involving the pump because it is largely absorbed in a single passage through the structure and the reflectance band of the Bragg stack is generally not wide enough to cover the pump wavelength. However, for in-well pumping, the overlap between the standing-wave pattern of the pump radiation and the locations of QWs needs to be optimized. The cavity enhancement factor $^{13}$ can be described as $\Gamma_{\text {cav }}=n_{\text {sc }} I_{\text {cav }} / n_{d} I_{0}$, where $n_{\mathrm{sc}}$ and $n_{d}$ are the refractive indices of the semiconductor and the diamond, respectively, and $I_{\text {cav }}$ and $I_{0}$ correspond to the intracavity field intensity and the incoming field intensity. The electrical field distribution of the pump has been calculated by determining the overlap integral of the pump field over the wells.

Similar arguments were used in Ref. 13. The enhancement factors $\Gamma_{\text {cav }}$ and $\Gamma_{\mathrm{RPG}}$ can be calculated based on our knowledge of the composition of the semiconductor structure used in this paper, and their dependencies as a function of wavelength are shown in Fig. 9. The set of dashed curves are the enhancement factors $\left(\Gamma_{\text {cav }}\right.$ and $\left.\Gamma_{\mathrm{RPG}}\right)$ at normal incidence, while the other set of solid curves are the factors at an incident angle of $27^{\circ}$. The curves shift to shorter wavelengths as the incident angle increases. Due to the antiresonant structure of the wafer, the main peak of the $\Gamma_{\mathrm{RPG}}$ curve does not overlap with the peaks of the $\Gamma_{\text {cav }}$ function. From the overlapping function $\left(\Gamma_{\mathrm{RPG}}\right)$ for the case of the $27^{\circ}$ incident angle, a wavelength of $847 \mathrm{~nm}$ would be optimum. However, it is far off a microcavity resonance and rather close to the laser wavelength, which will lead to strong absorption bleaching; hence it is not suitable for pumping. The cavity resonance at $840 \mathrm{~nm}$ corresponds still to a high value of $\Gamma_{\mathrm{RPG}}$ but remains very close to the laser wavelength. We are therefore forced to select our pump wavelength to be either another resonance of the microcavity or the second lower peak for RPG

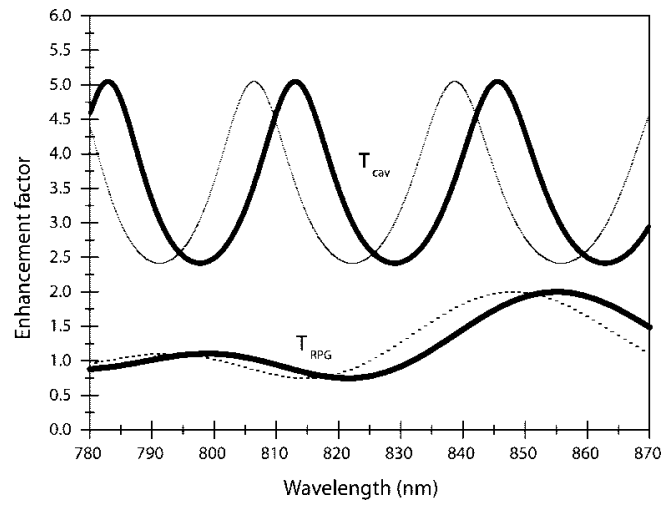

Fig. 9. Calculated values of the enhancement factors $\left(\Gamma_{\text {cav }}\right.$ and $\Gamma_{\mathrm{RPG}}$ ) as a function of wavelength for both normal incidence (dashed curve) and at an angle of $27^{\circ}$ (solid curve).

enhancement depending on which factor is dominant. For our current wafer structure, the microcavity enhancement is stronger than the enhancement by RPG structure. Hence the resonance at $806 \mathrm{~nm}$ is chosen for our pumping wavelength, where the average RPG factor $\Gamma_{\mathrm{RPG}}=0.868$ indicates a rather poor overlap between pump and QWs. Indeed, by modeling the pump electric field distribution, the absorption at each QW inside the current sample was also examined. It is found that three QWs located in the central part of the active region hardly absorb pump light. Those QWs will act as absorbers to the laser radiation. This could partly explain the relatively high internal loss of the laser $(\sim 3 \%)$ obtained in the gain measurement discussed above. Hence it would be advantageous in a future design to replace unpumped QWs with pump transparent spacers and possibly group two or more QWs at the antinode of the cavity standing wave to maintain their total number. This would not only reduce the internal loss of the laser but also increase the total pump absorption as $\Gamma_{\mathrm{RPG}}$ approaches its theoretical maximum limit of 2 .

The third aspect of the improvement of the overall performance of in-well pumping is to increase the absorption per QW. This could be achieved by widening the QWs. However, to maintain the quantum nature, this would require the walls of the wells to be higher. This is entirely feasible because the barrier bandgap is a free parameter for an in-well-pumped system.

\section{Conclusion and Outlook}

An output of $1 \mathrm{~W}$ at $850 \mathrm{~nm}$ has been demonstrated from an optically in-well-pumped VECSEL. This was achieved using a fiber-coupled laser diode emitting at $\sim 800 \mathrm{~nm}$ and reflecting the nonabsorbed power after one double pass back onto the gain medium. Laser gain has also been investigated demonstrating a logarithmic dependence on the pump intensity. The pump light was only absorbed in the QWs. It has been shown that the absorption of the pump light is influenced by a weak microcavity in the gain element, and therefore the wavelength of the pump source is cru- 
cial. First, the absorption can be further enhanced by optimizing the overlap integral of the pump field over QWs together with grouping QWs. Second, the absorption per QW as well as the number of QWs can be increased. Third, three to five additional recycling loops of the pump light will guarantee absorption of nearly all the pump power. This requires a high reflectivity for the Bragg mirror for both the laser and the pump light. Further investigations taking these suggestions into account are currently planned. It seems reasonable to expect that future systems would achieve output powers of several watts around $850 \mathrm{~nm}$ with good beam quality, similar to the system reported by emitting at $1000 \mathrm{~nm} .^{4}$

We thank David Burns from the Institute of Photonics at Strathclyde who pointed the possibility of in-well pumping out to us and John Roberts from the University of Sheffield for manufacturing the VECSEL. This project was partly funded by the Nuffield Foundation. Wei Zhang is supported by an Overseas Research Student scholarship.

\section{References}

1. W. J. Alford, Th. D. Raymond, and A. A. Allerman, "High power and good beam quality at $980 \mathrm{~nm}$ from a vertical external-cavity surface-emitting laser," J. Opt. Soc. Am. B 19, 663-666 (2002).

2. R. Haering, R. Paschotta, A. Aschwanden, E. Gini, F. MorierGenoud, and U. Keller, "High-power passively mode-locked semiconductor lasers," IEEE J. Quantum Electron. 38, 12681275 (2002).

3. M. A. Holm, D. Burns, A. I. Ferguson, and M. D. Dawson, "Actively stabilized single-frequency vertical-external-cavity AlGaAs laser," IEEE Photon. Technol. Lett. 11, 1551-1553 (1999).

4. J. Chilla, St. Butterworth, A. Zeitschel, J. Charles, A. Caprara, M. Reed, and L. Spinelli, "High power optically pumped semiconductor lasers," in Solid State Lasers XIII: Technology and Devices, R. Scheps and H. J. Hoffman, eds., Proc. SPIE 5332, 143-150 (2004).

5. J. E. Hastie, J. M. Hopkins, S. Calvez, C. W. Jeon, D. Burns, R. Abram, E. Riis, A. I. Ferguson, and M. D. Dawson, "0.5-W single transverse-mode operation of an 850-nm diode-pumped surface-emitting semiconductor laser," IEEE Photon. Technol. Lett. 15, 894-896 (2003).

6. J. E. Hastie, L. G. Morton, A. J. Kemp, M. D. Dawson, A. B. Krysa, and J. S. Roberts, "Tunable ultraviolet output from an intracavity frequency-doubled red vertical-external-cavity surface-emitting laser,” Appl. Phys. Lett. 89, 061114 (2006).

7. C. Salomon, J. Dalibard, W. D. Phillips, A. Clairon, and S. Guellati, "Laser cooling of cesium atoms below 3-mu-K," Europhys. Lett. 12, 683-688 (1990).

8. T. Kurosu and F. Shimizu, "Laser cooling and trapping of calcium and strontium," Jpn. J. Appl. Phys., Part 2 29, L2127L2129 (1990).

9. C. C. Bradley, J. J. McClelland, W. R. Anderson, and R. J. Celotta, "Magneto-optical trapping of chromium atoms," Phys. Rev. A 61, 053407 (2000).
10. M. Schmid, S. Benchabane, F. Torabi-Goudarzi, R. Abram, A. I. Ferguson, and E. Riis, "Optical in-well pumping of a vertical-external-cavity surface-emitting laser,” Appl. Phys. Lett. 84, 4860-4862 (2004).

11. Y. A. Chang, J. R. Chen, H. C. Kuo, Y. K. Kuo, and S. C. Wang, "Theoretical and experimental analysis on InAlGaAs/AlGaAs active region of 850-nm vertical-cavity surface-emitting lasers," J. Lightwave Technol. 24, 536-643 (2006).

12. L. A. Coldren and S. W. Corzine, Diode Lasers and Photonic Integrated Circuits (Wiley, 1995).

13. S.-S. Beyertt, M. Zorn, T. Kubler, H. Wenzel, M. Weyers, A. Giesen, G. Trankle, and U. Brauch, "Optical in-well pumping of a semiconductor disk laser with high optical efficiency," IEEE J. Quantum Electron. 41, 1439-1449 (2005).

14. A. Giesen, H. Hugel, A. Voss, K. Wittig, U. Brauch, and H. Opower, "Scalable concept for diode-pumped high-power solidstate lasers," Appl. Phys. B 58, 365-372 (1994).

15. M. Schmid, "VECSELs: an attractive new source in the nearIR,” Photonics Spectra 38, 58-64 (2004).

16. M. I. Muller, C. Karnutsch, J. Luft, W. Schmid, K. Streubel, N. Linder, S.-S. Beyertt, U. Brauch, A. Giesen, and G. H. Dohler, "Optically pumped vertical-external-cavity semiconductor thin disk laser with cw operation at $660 \mathrm{~nm}$," in Proceedings of the International Symposium on Compound Semiconductors (IOP, 2002), Vol. 174, pp. 427-430.

17. K. J. Knopp, D. H. Christensen, and J. R. Hill, "Vertical-cavity surface-emitting lasers with low-ripple optical pumping windows," IEEE J. Sel. Top. Quantum Electron. 3, 366-371 (1997).

18. M. J. Bohn and J. G. McInerey, "Resonant optical pumping of vertical-cavity surface emitting lasers," Opt. Commun. 117, 111-115 (1995).

19. M. Holm, "Vertical external cavity surface emitting lasers," $\mathrm{Ph} . \mathrm{D}$. thesis (University of Strathclyde, 2001).

20. Z. L. Liau, "Semiconductor wafer bonding via liquid capillarity," Appl. Phys. Lett. 77, 651-653 (2000).

21. S. McGinily and E. Riis, Department of Physics, University of Strathclyde, 107 Rottenrow, Glasgow G4 0NG, U.K., are preparing a manuscript to be called, "Optically pumped $850 \mathrm{~nm}$ VECSEL based on InAlGaAs material."

22. A. J. Kemp, G. J. Valentine, J. M. Hopkins, J. E. Hastie, S. A. Smith, S. Calvez, M. D. Dawson, and D. Burns, "Thermal management in vertical-external-cavity surface-emitting lasers: finite-element analysis of a heatspreader approach," IEEE J. Quantum Electron. 41, 148-155 (2005).

23. R. H. Abram, K. S. Gardner, E. Riis, and A. I. Ferguson, "Narrow linewidth operation of a tunable optically pumped semiconductor laser," Opt. Express 12, 5434-5439 (2004).

24. A. Yariv, Optical Electronics in Modern Communications (Oxford U. Press, 1991).

25. M. Kuznetsov, F. Hakimi, R. Sprague, and A. Mooradian, "Design and characteristics of high power (0.5-W CW) diodepumped vertical-external-cavity surface-emitting semicon-

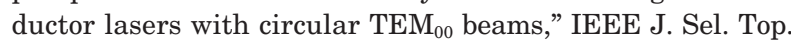
Quantum Electron. 5, 561-573 (1999).

26. M. Y. A. Raja, S. R. J. Brueck, M. Osinski, C. F. Schaus, J. G. McInerney, T. M. Brennan, and B. E. Hammons, "Resonant periodic gain surface-emitting semiconductor-lasers," IEEE J. Quantum Electron. 25, 1500-1512 (1989). 